\title{
El recuerdo y la emoción en la adquisición del hábito lector. Un estudio de caso*
}

\section{The memory and emotion in the acquisition of the reading habit. A case study}

\author{
Eva Álvarez Ramos
}

Universidad de Valladolid

evamaria.alvarez.ramos@uva.es

ORCID ID: https://orcid.org/0000-0001-7812-6592

\author{
Belén Mateos Blanco \\ Universidad de Valladolid \\ mariabelen.mateos@uva.es \\ ORCID ID: https://orcid.org/0000-0002-1283-1552
}

\section{Leyre Alejaldre Biel}

Columbia University

leyre.alejalldre@columbia.edu

ORCID ID: https://orcid.org/0000-0001-6805-0846

\section{Agustín Mayo-Iscar}

Universidad de Valladolid

agustinm@eio.uva.es

ORCID ID: https://orcid.org/0000-0003-0951-6508

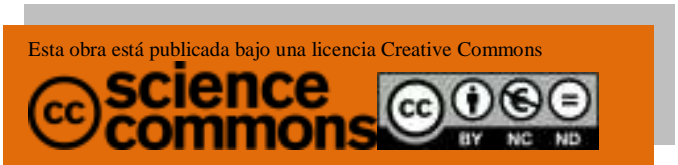

DOI: $10.17398 / 1988-8430.34 .293$

Fecha de recepción: 29/04/2021

Fecha de aceptación: 28/06/2021

Álvarez Ramos, E.; Mateos Blanco, B.; Alejaldre Biel, L., y Mayo-Iscar, A. (2021). El recuerdo y la emoción en la adquisición del hábito lector. Un estudio de caso. Tejuelo, 34, 293-322.

Doi: https://doi.org/10.17398/1988-8430.34.293

\footnotetext{
* Este trabajo se ha realizado en el marco del Proyecto de Investigación «Fractales. Estrategias para la fragmentación en la narrativa española del siglo XXI» (PID2019-104215GB-I00), financiado por el Ministerio de Ciencia e Innovación". Además, ha sido parcialmente apoyado por el Ministerio de Economía Español y Competitividad (MTM2017-86061-C2-1-P) y por la Consejería de Educación de la Junta de Castilla y León y Fondos FEDER (VA005P17 y VA002G18).
} 
Resumen: Las recientes investigaciones llevadas a cabo por la neurociencia aplicadas concretamente al ámbito educativo ponen de manifiesto cómo nuestro cerebro guarda con una mayor precisión y a un mayor largo plazo aquellos acontecimientos, saberes, experiencias que se relacionan directamente con una emoción (bien sea positiva, bien sea negativa). Pretendemos, a través de este estudio, descubrir si los recuerdos y emociones adscritos a la lectura en la infancia infieren directamente en la adquisición del hábito lector. Para ello se ha efectuado una investigación mixta (a lo largo de 6 cursos académicos) entre el alumnado de cuarto curso de las facultades de educación de la Universidad de Valladolid (Campus de Valladolid y de Segovia) matriculados en las asignaturas Literatura Infantil y Conocimiento del Entorno a Través de la Literatura Infantil. La intención es discernir y mostrar qué tipos de emociones y recuerdos afectan directamente a la lectura y si estos guardan o no una relación constatada con el hábito lector actual.

Palabras clave: hábito lector; neurociencia; neuroeducación; emoción; recuerdo.
Abstract: The recent research carried out by neuroscience applied specifically to the educational field shows how our brain saves with greater precision and in a longer term those events, knowledge, experiences that are directly related to an emotion (either positive, or negative). We intend, through this study, to discover if the memories and emotions ascribed to reading in childhood directly infer the acquisition of the reading habit. To this end, a mixed investigation has been carried out (over 6 academic years) among fourth-year students from the faculties of education of the University of Valladolid (Valladolid and Segovia Campus) enrolled in the subject of Children's Literature and Knowledge of the Environment Through Children's Literature. The intention is to discern and show what types of emotions and memories directly affect reading and whether or not they have a proven relationship with the current reading habit.

Keywords: reading habit; neuroscience; neuroeducation; emotion; memory. 
Without the stimulus and guidance of emotion, rational thought slows and disintegrates. The rational mind does not float above the irrational. It cannot free itself to engage in pure reason. There are pure theorems in mathematics, but no pure thoughts that discover them (Wilson, 1998, p. 113)

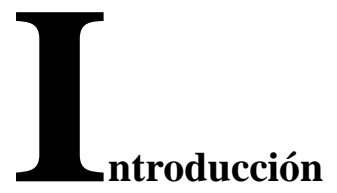

Las investigaciones en el ámbito de las ciencias del cerebro y, más concretamente, las relativas a la neurociencia cognitiva nos permiten aseverar que percibimos el mundo y lo que acontece "a través de los filtros emocionales del cerebro" (Mora, 2013a, p. 1). Hasta la fecha, el ámbito de las emociones y sus aledaños era considerado como superfluo y de una validez exigua frente a la superioridad de la razón. No se concebía el conocimiento, si no era ajeno al sentimiento. En los últimos años, los estudios sobre la emoción se han reactivado enérgicamente, uniéndose a la larga trayectoria ya existente (James, 1884; Marañón, 1920; Damasio, 1999; Le Doux, 1999; Mora, 2013a y 2013b).

Al hilo de estos avances, resulta interesante poner de manifiesto cómo interfiere la emoción en el desarrollo del hábito lector, más concretamente, si ubicamos los sentimientos en los recuerdos infantiles de lectura. Los fenómenos emocionales adscritos a la misma y a sus circunstancias (no solo entra en juego el texto en sí, sino todo el aparato derivado del hecho lector: compañeros, lugares, momentos...) inciden 
directamente en el gusto o disgusto lector, así como en los hábitos lectores adultos. Esta "implicación emocional del lector se revela como un componente intrínseco del proceso" (Sanjuán, 2014, p. 165). Aquellas experiencias plenas redundarán en la construcción de lectores, frente a las negativas que disminuirán su presencia, porque es, precisamente, ese el valor de la emoción,

una conducta que incluye todos los cambios que se producen en el cuerpo disparatados por un amplio rango de estímulos que vienen de todo cuanto rodea al individuo (o que también pueden producirse desde la evocación de la memoria de tales estímulos) y que indican recompensas (placer) o castigo (dolor) (Mora, 2013b, p. 65).

Toda emoción conlleva etimológicamente un cambio, que se ve reflejado física y mentalmente: la percepción de algo externo infiere en nosotros mutándonos (James, 1884). Estas conmociones físicas y anímicas no se quedan solamente en eso, estímulos pasajeros que llegan y desaparecen. Llevan aparejado un poso, un residuo que puede promover asociaciones negativas o positivas a los actos que han desencadenado esas emociones.

Los recuerdos, del mismo modo, nos permiten configurar lo que Bayard (2008) concibe como 'bibliotecas interiores' o lo que Devetach (2008) denomina 'textotecas internas': esos textos construidos "con palabras, historias, dichos, poemas, piezas del imaginario individual, familiar y colectivo" (pp. 37-38) y que, además se alimentan de emociones, puesto que estas, en definitiva, son las que sustentan dichos procesos de memoria (Mora, 2013b). Ese camino, que como lectores iniciamos en la infancia recorre una trayectoria marcada por "los libros que nos dieron a leer, por las tareas de lectura que tuvimos que abordar, por los modelos de lectores que conocimos, por el modo en que nuestras experiencias lectoras nos hicieron sentir" (Granado y Puig, 2015, p. 44).

La lectura no es un proceso mecánico en el que intervienen ojo, cerebro y texto (Smith, 1995). La lectura emociona y conmueve, expande el tiempo y el espacio (Bettleheim y Zelan, 2001). Cuando "in listening to poetry, drama, or heroic narrative, we are often surprised at 
the cutaneous shiver which like a sudden wave flows over us, and at the heart-swelling and the lachrymal effusion that unexpectedly catch us at intervals" (James, 1884, p. 196). El placer o desagrado que produzca en el lector provocará la configuración de un recuerdo que facultará o impedirá el trayecto lector. Inherente al ser humano, en su vertiente más epicúrea, es la búsqueda de placer. Desde los instintos más primarios a los más racionales, la demanda de felicidad es una constante en la vida humana. No existe placer impuesto, siempre es voluntario, empujado por un deseo, una necesidad de consecución.

La lectura, como placer, se relaciona directamente con el deseo (Caivano, 2001). Huye de la imposición, "sin deseo, las lecturas son mediocres y su destino inexorable es el olvido" (Salazar, 2005a, p. 23). Esta afirmación parece verse reflejada en la educación literaria actual, que parece más motivada por otro tipo de prácticas desviadas de la propia naturaleza del proceso lector (Sanjuán, 2014).

El hábito lector, uno de los conceptos centrales de este trabajo, aspira a erigirse como la prueba fehaciente de que existe una relación constatable entre las emociones y los recuerdos que la lectura provoca en el lector y en sus rutinas lectoras. Avivar el interés por leer está intrínsecamente vinculado a la creación de un hábito porque "el deseo de leer es el factor más poderoso para generar hábitos de lectura y nace de asociar esta actividad al placer, a la satisfacción, a la sensación de logro y al entretenimiento" (Salazar, 2005a, p. 23). En la consecución de este fin, entran en juego mediadores como la familia y el profesorado, representantes ambos de dos ámbitos (tantas veces enfrentados): el académico y el vernáculo o social. El círculo más cercano del lector influye directamente en su interés, pasión y motivación por la lectura, así como en el desarrollo de su hábito lector. El contexto donde se desarrolla la lectura (escolar/académico o personal) y el objetivo de la misma (aprendizaje o placer) también condicionan las emociones del lector y la construcción de un hábito óptimo. De hecho, incide, en gran medida, la motivación, pudiendo ser favorecida esta actitud en el contexto familiar (Mora- Figueroa, Galán y López-Jurado, 2016). 
Los menores con un hábito lector más desarrollado asocian la lectura al ocio y, además, comparten esta afición con alguien de su familia, así lo señalan Strommen y Mates (2004), que destacan que: "Readers learned from family, or other members of their social circle, that reading can be an entertaining, diverting, enjoyable, sociable, and, therefore, worthwhile activity" (p. 193). De lo que se puede concluir, que el vínculo emocional que la familia transfiere al acto de leer favorece el hábito de los menores (Martínez y Torres, 2019) y apoya la hipótesis de la asociación de emociones positivas para la creación de lectores:

En los primeros contactos con la lectura y, en su posterior desarrollo, algunos estudios relacionan la motivación lectora con la implicación de las familias en este hábito; otros estudios la vinculan con el ambiente literario del hogar; y otros la condicionan a las acciones intencionadas realizadas por la familia para fomentar la lectura (p. $105)$.

Al entorno familiar hay que añadir el impacto del ámbito escolar y, más concretamente, la influencia que puede tener los maestros en los futuros lectores. Las investigaciones llevadas a cabo por Martínez y Torres (2019); Díaz-Plaja y Prats (2013) o Sanjuán (2014) señalan la importancia en la formación y capacitación del profesorado para incentivar el hábito lector del alumnado, “de poco sirven las políticas institucionales de fomento de la lectura y de las bibliotecas escolares sin un profesorado sólidamente formado para ello" (Granado, 2013, p. 112).

El objetivo (placer o fines académicos) también influye en las emociones que pueden conmocionar al lector y que interfieren en el recuerdo, que se asociará a la lectura y redundará en el hábito. Así lo señalan Mata (2010) y Sanjuán (2011) que sostienen que leer para cumplir objetivos escolares impacta negativamente en la emoción y el disfrute, nos aparta del placer del acto y reduce la lectura a una tarea de estudio.

Parecía necesario poder investigar, hasta qué extremo el recuerdo derivado de una sensación lectora incidía en el desarrollo del 
hábito lector. Y, todo ello, enfocado a un colectivo, pieza clave en la mediación lectora, como es el de los maestros en formación.

Pasamos, ahora, a exponer el objetivo principal de esta investigación: demostrar el influjo del recuerdo y de la emoción en el hábito lector. Para ello se han delimitado varias cuestiones de investigación, que nos facultarán la obtención de los resultados. Para la elaboración de las mismas se han tenido presentes varias de las características demandas por Bloomberg (2016), Silverman (2013) y Farrugia et al. (2010): viabilidad, relevancia especificidad, interconexión y ausencia de ambigüedad.

\section{Metodología}

\section{1. Participantes}

La muestra ha estado conformada por 326 estudiantes pertenecientes al Grado de Educación Primaria, Grado de Educación Infantil y Programa de Estudios Conjuntos de Grado en Educación Primaria y Grado en Educación Infantil de las facultades de educación de la Universidad de Valladolid ubicadas en los campus de Valladolid y Segovia. Todos los sujetos cursaban la asignatura de $4 .^{\circ}$ curso Literatura Infantil o Conocimiento del Entorno a través de la Literatura Infantil. Los datos han sido recogidos consecutivamente entre los cursos académicos 2015/2016 y 2020/2021.

\section{Tabla 1}

Distribución por curso

\begin{tabular}{lcccc}
\hline Curso & n & \% & IC95 \% & IC95 \% \\
\hline $2015 / 2016$ & 45 & 13.8 & 4.8 & 8.4 \\
$2016 / 2017$ & 31 & 9.5 & 2.9 & 5.7 \\
$2017 / 2018$ & 27 & 8.3 & 2.6 & 5.3 \\
$2018 / 2019$ & 48 & 14.8 & 5.2 & 9.0 \\
$2019 / 2020$ & 72 & 22.1 & 8.9 & 13.7 \\
$2020 / 2021$ & 103 & 31.5 & 14.9 & 22.1 \\
\hline
\end{tabular}

Fuente: Elaboración propia 
La población estudiada muestra una proporción disímil en cuanto a la distribución de género, contando con un $79.1 \%$ de mujeres, frente a un $20.9 \%$ de hombres.

\section{Tabla 2}

Distribución por sexo

\begin{tabular}{lcccc}
\hline Sexo & $\mathbf{n}$ & \% & IC95 \% & IC95 \% \\
\hline Femenino & 258 & 79.1 & 27.3 & 33.5 \\
Masculino & 68 & 20.9 & 9.4 & 14.7 \\
\hline
\end{tabular}

Fuente: Elaboración propia

La presencia femenina es representativa de su posicionamiento a nivel nacional. Datos extraídos del Ministerio de Educación y Formación Profesional (2019, p. 45) señalan que la distribución porcentual, según el sexo, del profesorado de Educación Primaria se encuentra en un $81.4 \%$ de mujeres y un $18.6 \%$ de hombres. Mientras que la de Infantil alcanza un $97.6 \%$ de mujeres frente a un $4 \%$ de hombres. La diferencia genérica es considerable.

\section{2. Instrumento}

Para llevar a cabo la investigación se utilizó un cuestionario creado ad hoc para el desarrollo de la misma y validado por los investigadores implicados. El instrumento se halla divido en varias dimensiones: una primera destinada a la obtención de datos personales y académicos, para poder acceder al perfil de los informantes. A través de ella conseguimos los datos relativos al sexo que permiten, cruzando informaciones, dar respuesta a la última cuestión planteada: ¿En todos los datos extraídos se puede ver la influencia de la variable sexo?

Una segunda dimensión enfocada a las biografías lectoras, en la que se extrae información relacionada con el recuerdo y la emoción. Engloba preguntas sobre las primeras lecturas realizadas, acerca de los cuentos o libros que recuerdan de la infancia, de sus personajes y libros favoritos u odiados, las sensaciones vinculadas con la lectura y la evocación de la lectura en voz alta por parte del docente. También se informa sobre el primer libro que se recuerda haber leído por placer y la edad en la que se leyó. Respondemos así a las preguntas 1, 2 y 3: ¿Las 
sensaciones infantiles de lectura están relacionadas con la frecuencia lectora? ¿El recuerdo de lectura en voz alta en la niñez por parte del maestro afecta al gusto lector? ¿Incide la edad de lectura por placer en la frecuencia lectora?

\section{Tabla 3}

Extracto preguntas cuestionario dimensión 2

\begin{tabular}{lc}
\hline Pregunta & Respuesta \\
\hline ¿Qué cuentos, poemas o canciones infantiles recuerdas? & Abierta \\
¿Cuáles son los primeros libros que recuerdas haber leído? & Abierta \\
¿Recuerdas alguna sensación relacionada con la lectura? & Abierta \\
¿Las sensaciones recordadas son positivas o negativas? & Cerrada \\
¿Recuerdas si tus profesores te contaban o leían cuentos? & Cerrada \\
¿Qué recuerdos y sentimientos tienes hacia esa lectura en voz & \\
alta? & Abierta \\
¿Cuál era tu libro favorito? ¿Por qué? & Abierta \\
¿Qué cuento clásico o libro te gustaba menos? ¿Por qué? & Abierta \\
¿Cuál es el primer libro que recuerdas haber leído por placer? & Abierta \\
¿Qué años tenías? & Cerrada \\
\hline
\end{tabular}

Fuente: Elaboración propia

Finalmente, accedemos a una tercera dimensión, reservada para evaluar los hábitos y gustos lectores actuales (coincidentes con el momento en el que se llevó a cabo la encuesta), con interrogantes como la lectura en la actualidad y el grado dentro del hábito lector. Con las respuestas de esta sección contribuimos a informar de las cuestiones 1 y 3 de la investigación: ¿Las sensaciones infantiles de lectura están relacionadas con la frecuencia lectora? ¿Incide la edad de lectura por placer en la frecuencia lectora?

\section{Tabla 4}

Extracto preguntas cuestionario dimensión 3

\begin{tabular}{lc}
\hline Pregunta & Respuesta \\
¿Lees ahora literatura infantil? & Cerrada \\
¿Lees en la actualidad? & Cerrada \\
¿Con qué frecuencia? & Cerrada \\
\hline
\end{tabular}

Fuente: Elaboración propia 
Se combinan respuestas cerradas, dicotómicas o tricotómicas, que se acompañan de abiertas para especificar, contextualizar y ampliar la información vertida.

La recogida de la información se ha realizado manteniendo siempre las mismas pautas. La actuación a priori se justifica por el interés de los investigadores en no influir en las respuestas de los encuestados.

\section{3. Procedimiento}

El procedimiento de recogida de datos ha sido homogéneo en todos los cursos académicos investigados. El muestreo fue intencional. Los cuestionarios se aplicaron dentro del horario académico de las asignaturas reseñadas. Se realizó en el primer semestre de los cursos referidos. Se presenta el instrumento el primer día, en el que se imparte la asignatura, y se realiza presencialmente y en papel. Los responsables supervisan la aplicación del cuestionario y en todo momento se garantiza el anonimato y la confidencialidad de los estudiantes. La participación fue totalmente voluntaria.

Para mantener la privacidad y el anonimato, así como para el análisis y la presentación de los resultados, se ha concedido a cada sujeto un código alfanumérico identificativo, en el que se indica el número de encuestado, el sexo y el curso académico:

\section{Tabla 5}

Leyenda del código identificativo de cada participante

\begin{tabular}{ccc}
\hline Número de encuestado & Sexo & Curso académico \\
\hline & & $15 / 16$ \\
n. $^{o}$ & M: mujer & $16 / 17$ \\
& H: hombre & $17 / 18$ \\
& & $18 / 19$ \\
& & $19 / 20$ \\
\hline
\end{tabular}

Fuente: elaboración propia 


\section{4. Análisis de datos}

Se resumieron las variables numéricas utilizando medianas $(\mathrm{m}$. y rangos intercuartílicos (R. I.) y las cualitativas utilizando porcentajes. Hemos obtenido intervalos de confianza al $95 \%$ (IC95\%) para los correspondientes porcentajes poblacionales. Para contrastar la no asociación entre variables cualitativas hemos utilizado el test chicuadrado y para contrastar la igualdad de localización de dos grupos en una variable numérica el test de Mann-Withney-Wilcoxon. Hemos representado en un diagrama de barras categorizado la relación entre dos variables cualitativas. Se consideraron como estadísticamente significativos valores de $\mathrm{p}$ menores que 0.05 . Los análisis se llevaron a cabo utilizando el paquete estadístico R v4.0 [R Core Team (2020). R: A language and environment for statistical computing. R Foundation for Statistical Computing, Vienna, Austria. URL https://www.Rproject.org/].

Para el análisis de los datos cualitativos se ha utilizado el software MAXQDA, que nos ha permitido la representación gráfica de los datos así como la concomitancia entre las opiniones vertidas.

\section{5. Resultados}

Antes de comenzar a desglosar los datos obtenidos que den respuesta a las preguntas de investigación, es conveniente mostrar cuáles son los niveles de lectura actuales de los sujetos encuestados y en qué grado han desarrollado el hábito.

\section{Tabla 6}

Distribución por perfil lector

\begin{tabular}{lcccc}
\hline Lector & $\mathbf{n}$ & \% & IC95 \% & IC95 \% \\
\hline no & 74 & 22.7 & 7.0 & 10.8 \\
sí & 252 & 77.3 & 29.4 & 36.0 \\
\hline
\end{tabular}

Fuente: Elaboración propia

Puede observarse que la diferencia entre lectores y no-lectores es bastante significativa (algo más de un quinto afirman no leer en la actualidad). No es un dato que se adscriba unívocamente a los alumnos 
en formación de esta investigación, sino que puede ser extrapolado al oficio de maestro en general. Las investigaciones llevadas a cabo por Benevides y Peterson (2010), Cremin et al. (2008) o Romero (2007), entre otros, ponen el punto de mira en la carencia del personal activo, mientras que las realizadas por Granado (2014), Granado y Puig (2015), Colomer y Munita (2013); Díaz-Plaja y Prats (2013); Sanjuán (2014); Munita (2012), Applegate y Applegate (2004) o Gupta (2004) se centran en el colectivo que nos atañe.

El peligro de la (no) lectura actual, ya atisbado por Mendoza y Díaz (2005), aumenta exponencialmente cuando nos centramos en futuros docentes de Educación Primaria e Infantil. Los maestros, como mediadores o "estímulo[s] externo[s] que acompañará[n] al aprendiz en el encuentro transgeneracional con el folclore y su cultura" (Romero y Álvarez, 2018, p. 101) son una pieza clave en la construcción de la identidad lectora, evocada por Chartier y Bourdieu (1985). Es de cumplida obligación que los maestros provean a los discentes de deseos, ejemplos y razones que estimulen su apetito lector, hecho difícilmente conquistable, si el profesor no es un modelo de lectura (Granado y Puig, 2015). No es este, sin embargo, el objeto de la investigación, pero no podíamos dejar de reseñarlo por la importancia que tiene en la educación literaria.

Los lectores habituales destacaban por encima de los demás. Casi la mitad de los encuestados (44.5\%) afirmó que leía con asiduidad. Apenas un $5 \%$ se consideraba lector empedernido, siendo superados por los no-lectores $(22.7 \%)$, que se encontraban también por encima de los que reconocían leer de vez en cuanto (18.1\%). 


\section{Figura 1}

Frecuencia lectora

\section{Frecuencia lectora}

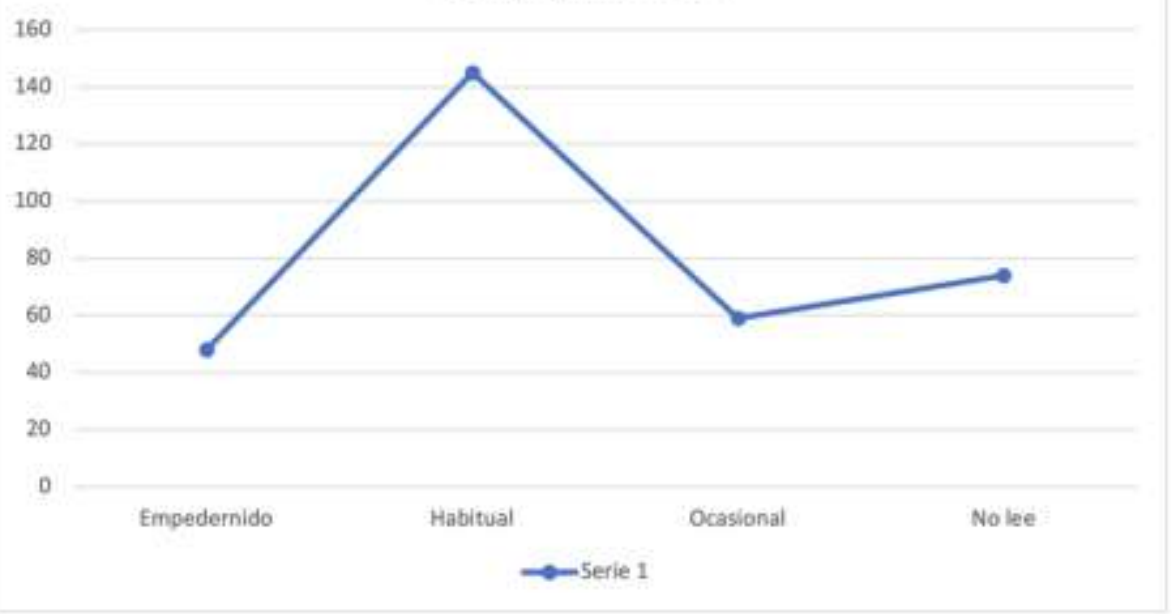

Fuente: elaboración propia

Para facilitar y simplificar el estudio de los datos, consideramos a los no-lectores como lectores ocasionales (sería imposible sostener que no se lee nada). Esta unificación se ve justificada por el hecho de que muchos de los encuestados solo consideran las prácticas de lectura activas (conscientes y voluntarias) (Ribera y Romero, 2020), aquellas que llevan aparejado un libro (un ente físico y analógico), así como una catalogación académica u ociosa, pero no recapacitan sobre las prácticas vernáculas (Camitta, 1993; Barton y Hamilton, 1998), las que se producen (con menos consciencia), al margen de las instituciones académicas y de lo más tradicional. Relacionadas con las nuevas generaciones de lectores y escritores (Lea y Street, 2006; Carlino, 2006), se inscriben en una sociedad claramente tecnológica, que ha mutado desde la médula sus medios y sistemas de comunicación (Piscitelli, 2002).

A los que respondieron afirmativamente se les permitió catalogar su hábito mediante tres variables (ocasional, habitual o empedernido). Nos parecía idóneo y necesario poder evaluar el grado o 
la frecuencia lectora de dichos sujetos a la hora de cruzar la incidencia con otras variables.

Tabla 7

Distribución por frecuencia del hábito lector

\begin{tabular}{lclcc}
\hline Frecuencia & n & \% & IC95 \% & IC95 \% \\
\hline empedernido & 48 & 14.7 & 4.3 & 7.4 \\
habitual & 145 & 44.5 & 15.6 & 20.9 \\
ocasional & 133 & 40.8 & 17.6 & 23.8 \\
\hline
\end{tabular}

Fuente: Elaboración propia

El porcentaje, por tanto, de lectores ocasionales se ve aumentado, alcanzando casi un $41 \%$ de los sujetos. Aunque esta categoría forme parte de los considerados como lectores, no deja de sorprender el elevado número de maestros en formación que afirman leer de vez en cuando. Asentados estos datos, pasamos a exponer los resultados de la investigación.

\section{Lectores actuales en función de las sensaciones de lectura recordadas}

Un $83 \%$ de los que dijeron tener una sensación positiva relacionada con la lectura leen en la actualidad, frente al $60.2 \%$ de los que confesaron una sensación negativa $(\mathrm{p}<0.001)$.

\section{Tabla 8}

Distribución por sensaciones de lectura

\begin{tabular}{lllcc}
\hline Sensación & $\mathbf{n}$ & \% & IC95 \% & IC95 \% \\
\hline negativa & 88 & 27.0 & 8.5 & 12.6 \\
positiva & 238 & 73.0 & 28.2 & 34.8 \\
\hline
\end{tabular}

Fuente: Elaboración propia

Las impresiones positivas recordadas y relacionadas con la lectura $(73 \%)$ superan notablemente a las negativas $(27 \%)$. Guardan una estrecha relación con el ámbito cercano y social del niño, principalmente, el familiar y el escolar -figura 2-. Los porcentajes extraídos, nos obligan a reflexionar, una vez más, sobre la pérdida del hábito lector según aumenta la edad y según van haciendo acto de presencia las prácticas letradas y académicas literarias. Hecho reforzado 
por las sensaciones negativas descritas por los encuestados, cuando rememoran sus lecturas escolares tales como: obligación, ansiedad, aburrimiento, deberes, no-gusto, pereza, falta de entusiasmo.

\section{Figura 2}

Sensaciones recordadas relacionadas con la lectura

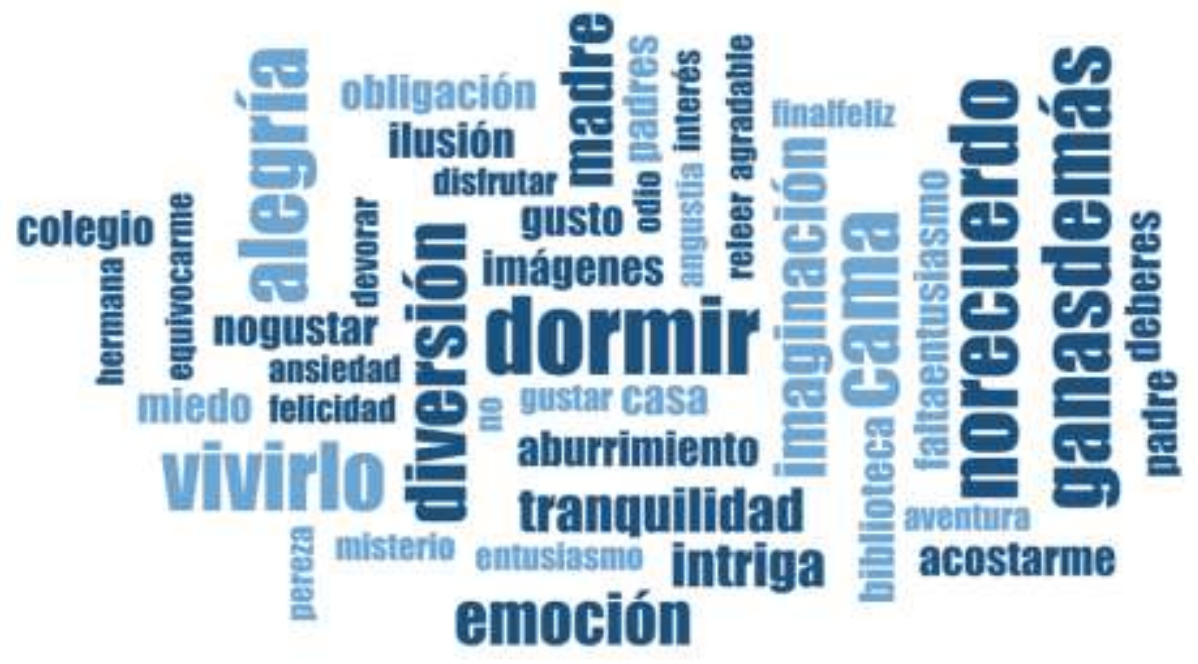

Fuente: Elaboración propia

Las experiencias literarias pedagógicas desembocan, antitéticamente, en resultados poco o nada formativos (Sanjuán, 2011), precisamente por ese cariz académico que emborrona la emoción y entorpece el disfrute, "se produce así una contradicción entre los propósitos declarados y las tareas escolares, de modo que la literatura, lejos del vitalismo y la transgresión que se le asigna, queda reducida a una simple materia de estudio" (Mata, 2010, p. 112). No ya solo por ser considerada contenido de aprendizaje, sino que la sensación se ve agravada por el apelativo 'obligatoria' y por la falta de empatía con el discente, al proporcionarle textos poco o nada atractivos. Así lo demuestra la información vertida por alguno de los encuestados:

"Me costaba bastante leer estos libros, eran deberes del colegio". $(15 \mathrm{H} 15 / 16)$

"Sentía que fuera un castigo porque realmente el argumento no me interesaba lo más mínimo". (54M16/17) 
"Leídos por obligación sin ningún entusiasmo". (148M18/19)

"Todas las sensaciones malas, las lecturas eran obligatorias". (287H20/21)

Pero la mayoría de las emociones aprehendidas son de corte positivo, cuando es el ambiente familiar el que entra en juego. Hemos optado por agruparlas en varias categorías que nos permitan profundizar en los sentimientos adscritos a la lectura.

\section{Figura 3}

Distribución por grado de sensaciones positivas y categorías de relación

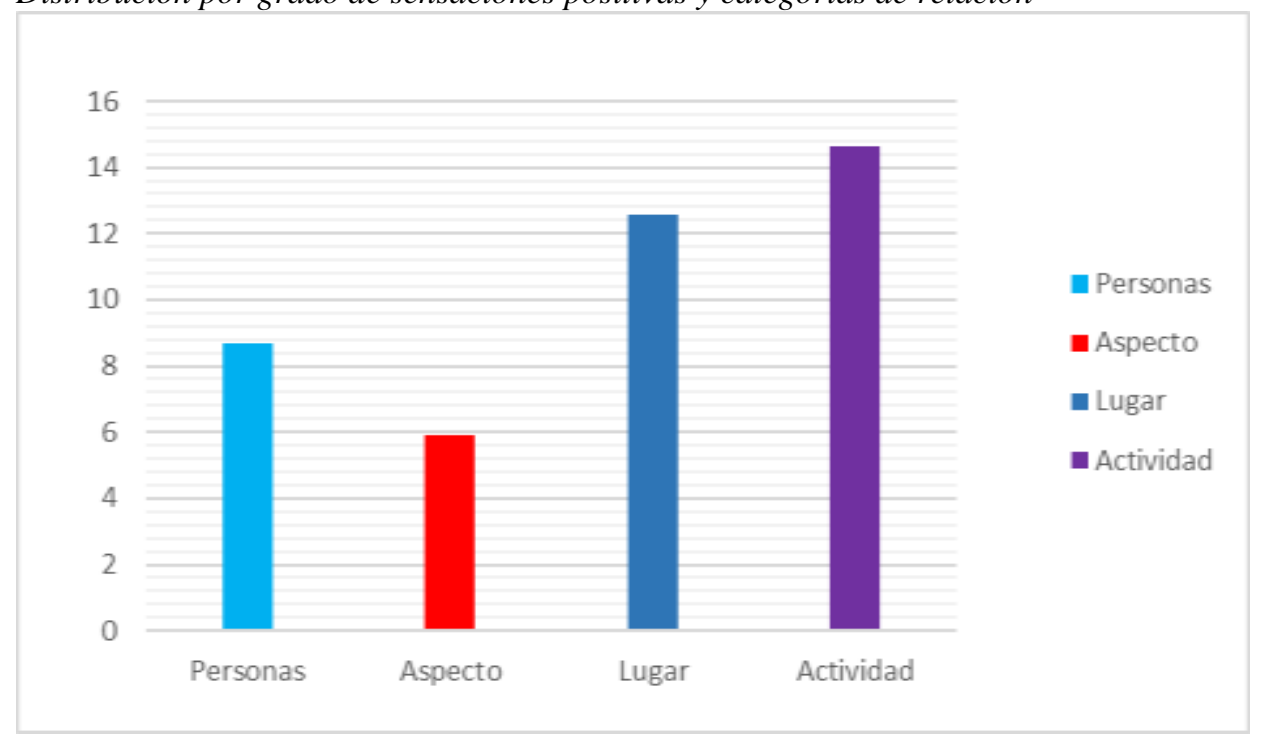

Fuente: Elaboración propia

Describen el recuerdo como una sensación agradable, que les proporciona paz, que les tranquiliza, que les hace viajar o ponerse en la piel del protagonista. Las personas más cercanas, como padres y hermanos, se relacionan también con el hecho lector. Bien por su actividad como mediadores (también hay recuerdos para los maestros y bibliotecarios) bien por sus tiempos de lectura compartidos, tal es el caso de los hermanos. El aspecto de los libros: portadas, tamaño, imágenes, incluso olores o texturas... permanecen también en la retina de los encuestados. Los lugares donde se leía y la actividad que se 
llevaba a cabo al tiempo o con posterioridad son enlazados, igualmente, con el recuerdo lector. Así, traen a la memoria meriendas, camas, vacaciones..., junto a actividades como comer, descansar o el tiempo previo al sueño (esa lectura antes de dormir). Este tipo de emociones caseras, despiertan en el cerebro una sensación placentera que contribuye a reforzar el gusto por la lectura. Extraemos a continuación alguno de estos recuerdos:

"En la cama, antes de acostarme, sensación de paz y tranquilidad" (3M15/16).

"Leer tumbado en la alfombra los días de lluvia, me da tranquilidad y sentido hogareño" (55H16/17).

"El no poder parar de leer, eran momentos de lectura justo antes de dormir en compañía de mis padres" (103H17/18).

"Leer los libros con mi madre y mi hermano tumbados en la cama, generalmente por la noche antes de dormir" (121H18/19).

"Recuerdo que por aquella época me leía los libros en un día" (128M18/19).

Estamos ante un cúmulo de "experiencias emocionales, que sin aflorar conscientemente, provocan en el lector 'chispazos' que le llevan a apreciar o rechazar (placer o disgusto) lo que lee" (Mora, 2020, p. 110-111).

Si prestamos atención al hábito lector, un $63.7 \%$ de los que mostraron una sensación positiva son lectores habituales o empedernidos frente al $43.1 \%$ de los que mostraron una sensación negativa. Es decir, las emociones positivas inciden directamente en la frecuencia del hábito lector.

\section{Lectores actuales en función de la lectura de cuentos por parte del maestro}

Más allá de los datos negativos de lectura en el colectivo de los maestros, señalado más arriba, sí parece establecerse como una práctica positiva y común en el aula, la lectura en voz alta en las etapas de infantil y primaria. Independientemente, de que hablemos de narración oral, defendida por Stewig (1980) y Pellowski (1987), o nos enfrentemos a una lectura en voz alta, estamos ante una actividad 
esencial en aula (Routman, 2000) que no solo ofrece modelos de lectura, sino que desarrolla actitudes positivas ante la misma a la par que deja, como demuestran los datos, recuerdos imborrables en la mente (Beuchat, 2013). Es vital la relación que integra armónicamente al libro, a quien lee y al que escucha (Fox, 2008).

Más de la mitad de los encuestados recuerdan con avidez la voz del maestro en el aula, frente a un $40 \%$ que afirma no haber recibido nunca interacción alguna. Es posible, que sí se llevara a cabo, pero no ha permanecido entre sus recuerdos infantiles.

\section{Tabla 9}

Distribución por recuerdos de lectura en voz alta del maestro

\begin{tabular}{llccc}
\hline $\begin{array}{l}\text { Lectura en } \\
\text { voz alta }\end{array}$ & $\mathbf{n}$ & \% & IC95 \% & IC95 \% \\
\hline no & 127 & 39.0 & 12.7 & 17.4 \\
sí & 199 & 61.0 & 24.6 & 31.2 \\
\hline
\end{tabular}

Fuente: Elaboración propia

Un $84.4 \%$ de los que dijeron que los profesores les leían cuentos leen en la actualidad, frente al $66.1 \%$ que leen entre aquellos a los que no les leían cuentos $(\mathrm{p}<0.001)$. No se encontró relación estadísticamente significativa entre que los profesores les hubieran leído cuentos y ser un lector habitual o empedernido. Hecho que no niega el valor de esta actividad por la cantidad de beneficios que se desprenden de ella, aunque el resultado de la investigación no haya sido significativo.

\section{Lectores actuales en función de la edad de primera lectura placentera}

Otra de las cuestiones que nos planteábamos guarda una estrecha vinculación con las prácticas letradas académicas y la relación que, creemos, se establece entre el placer y disfrute de lo leído y la adquisición del gusto lector. La emoción estalla no ya solo unida a un recuerdo, una imagen, una sensación externa al hecho lector, sino que se circunscribe dentro del texto. Esa experiencia individual y personal 
que rezuma de la relación entre literatura y lector y que posibilita la transformación de este último a través de lo leído (Sanjuán, 2011).

No todos tenían consciencia o recordaban esa primera lectura placentera. Algunos, ni siquiera creían haberla disfrutado. Un total de 269 sujetos, es decir, casi un $75 \%$ dieron respuesta a esta cuestión, lo que conlleva que cuando se imprime en el cerebro una sensación positiva, esta es registrada y almacenada a largo plazo.

Optamos por una taxonomía cuatripartita, obteniendo grupos de edades lectoras coincidentes con los ciclos escolares

- Hasta once años, abarcábamos así toda la etapa de Educación Primaria. (1)

- De doce a quince años, franja que coincide con la Educación Secundaria Obligatoria. (2)

- De dieciséis a dieciocho, representando el Bachillerato. (3)

- Mayores de dieciocho, grupo reflejo de la Educación Superior. (4)

\section{Tabla 10}

Rangos de edad primera lectura deseada

\begin{tabular}{lrrrc}
\hline $\begin{array}{l}\text { Rango de } \\
\text { edad }\end{array}$ & n & \% & IC95 \% & IC95 \% \\
\hline 1 & 139 & 51.7 & 16.2 & 21.8 \\
2 & 79 & 29.4 & 11.0 & 16.5 \\
3 & 19 & 7.1 & 2.4 & 5.8 \\
4 & 32 & 11.9 & 4.7 & 9.1 \\
\hline
\end{tabular}

Fuente: Elaboración propia

Las lecturas placenteras se producen en un grado considerable durante la educación primaria (más de la mitad de los encuestados), etapa de descubrimiento de la lectura y que, todavía (en cierto modo), permite un acceso a la misma aséptico y libre de academicismos, hecho que ya no sucede en secundaria (casi un $30 \%$ ) y mucho menos en bachillerato (menos de un $10 \%$ ).

Del mismo modo, los porcentajes de lectores actuales aparecen inversamente relacionados con la edad a la que leyeron por primera vez 
de forma placentera. De esta forma, estos porcentajes de lectores actuales disminuyeron en nuestra muestra al recorrer en sentido ascendente los grupos de edad de primera lectura placentera: $86.3 \%$, $75.9 \%, 78.9 \%, 59.4 \%(\mathrm{p}<0.001)$

\section{Figura 4}

Lector actual según edad primera lectura

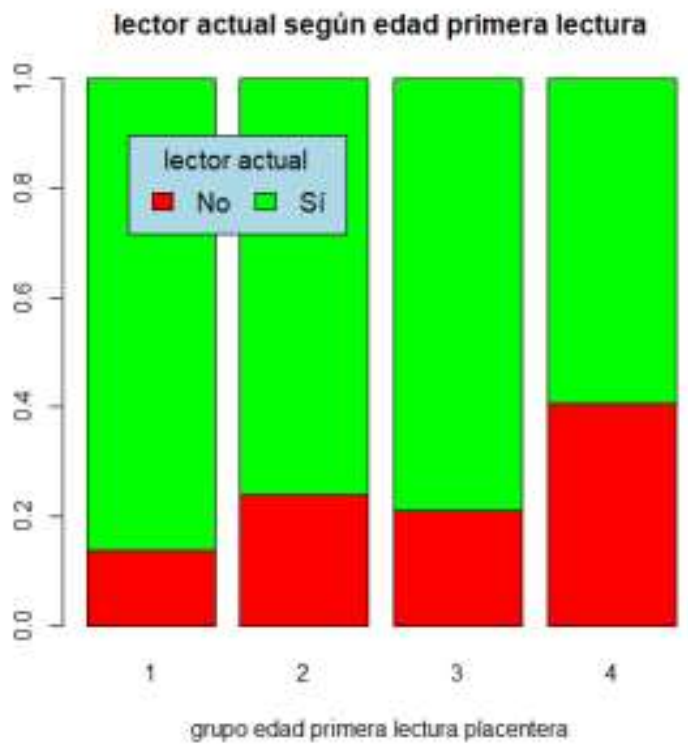

Fuente: Elaboración propia

Este último dato extraído resulta muy alentador, puesto que demuestra que la sonada pérdida de lectores en el período comprendido entre los 12 y los 16 años es recuperable si existe ese poso afectivo adquirido durante la Educación Primaria. Aquellos encuestados que reconocen haber adquirido el gusto lector de forma temprana, se constituyen después como grandes y ávidos lectores (habituales o empedernidos). Se valora la literatura, de manera general, por el placer que produce (Wigfield y Guthrie, 1997; Applegate y Applegate, 2004; Sainsbury y Schagen, 2004; Sanjuán, 2011; Granado y Puig, 2015). 
Por otra parte, los datos obligan a plantearse muy seriamente la educación literaria en primaria, puesto que los resultados demuestran su efectividad a largo plazo.

\section{Incidencia de la variable 'sexo'}

Finalizamos con la última pregunta de investigación. El grueso de los que empezaron a leer más tarde son mujeres $[\mathrm{m}=6($ r.i. $=12.5)]$ frente a los varones $[4(6.25)](\mathrm{p}<0.001)$. Es cierto que este estudio se caracteriza por una presencia femenina que supera en un alto porcentaje a la masculina. Reseñable, asimismo, es el hecho de que de los 139 individuos que recuerdan haber leído por placer durante los estudios de primaria, solo un $19 \%$ son hombres. Estos datos no enmascaran la supremacía de la presencia femenina en los grupos 3 y 4 (bachillerato y estudios superiores). Quizá se relacione con la incidencia que los estereotipos de género tienen mayoritariamente sobre el género femenino. Las investigaciones llevadas a cabo por Bian, Leslie y Cimpian (2017) afirman que su impacto afecta a largo plazo a las mujeres e impide que tengan confianza en sí mismas y tomen decisiones por sí solas. Esa tendencia a no salirse de lo pautado puede llevarlas a aceptar lo que se les impone, entre ellas las lecturas infantiles o adolescentes. De ahí, que la toma de decisión y elección de lectura por placer se retrase.

No apareció evidencia de relación estadísticamente significativa entre el sexo y las otras respuestas a las variables relacionadas con la tipología de lector. Las emociones, por tanto, afectan e inciden igualmente en hombres y en mujeres, contradiciendo la idea tradicional patriarcal de la emotividad femenina, frente a la rudeza o insensibilidad masculina.

\section{Conclusiones}

Los datos extraídos aportan bastante luz al panorama lector. Para comenzar estas conclusiones, es necesario reincidir en las carencias lectoras del profesorado en formación por toda la problemática futura que se deviene de la ausencia de hábito lector (Mendoza y Díaz, 2005; 
Granado y Puig, 2014) y que acaba generando un círculo vicioso (casi ad nauseam) de mediadores desinformados que no educan lectores. Los números extraídos de esta investigación, así como los mostrados por otros estudios, hacen saltar la alarma del estado actual de la formación inicial de docentes en particular (Granado, 2014; Granado y Puig, 2015; Colomer y Munita, 2013; Díaz-Plaja y Prats; 2013; Sanjuán, 2014; Munita, 2012; Applegate y Applegate, 2004 o Gupta, 2004) y, en general, de la educación literaria a lo largo de la vida. Habría que replantearse, vista la importancia que el recuerdo y la emoción adquieren en edades tempranas, qué tipos de mediación literaria se desarrollan y cuáles de ellos son los más efectivos. Es necesaria, pues, una revisión profunda de los pilares que sustentan la literatura y lo literario en el ámbito académico.

La demanda anterior enlaza directamente con el fehaciente dato (cuantitativo y cualitativo) de las impresiones positivas y negativas recordadas y relacionadas con la lectura. El cerebro infantil imprime la emoción del recuerdo y la ancla a las lecturas infantiles, lo que origina lectores habituales cuando la movere es positiva, o sujetos que rechazan todo lo relacionado con la lectura por haberse producido una sensación negativa. Paradójicamente, la mayoría de estas emociones adversas provienen del ámbito escolar, es decir, son las prácticas académicas las que las generan (Sanjuán, 2011). Calificativos como 'obligatorio' o 'escolar' inciden profundamente en el rechazo lector, a esto, podemos añadirle, la mala selección de lecturas, hecho que refuerza, una vez más, la falta de formación docente. La escuela no debería ser el problema, sino todo lo contrario.

En otro orden de cosas, las sensaciones positivas provienen, generalmente, del ámbito familiar, del espacio vernáculo, separado ágilmente por el niño, que sabe discernir entre uno y otro. Los recuerdos, grabados a fuego en las mentes infantiles, hacen revivir sensaciones placenteras, amables y tiernas, que los sujetos rememoran con fruición y enlazan (y aquí radica su importancia) con la lectura. Como hemos demostrado, no solo los libros (esas historias narradas, esos mundos paralelos que se generan en cada lectura (Bettleheim y Zelan, 2001)- emocionan. Turba, de la misma manera, el ambiente en el 
que se lleva a cabo. Es vital destacar la clara incidencia de la emoción y el recuerdo en la adquisición del hábito por la lectura. Porque, tal y como hemos visto, en la infancia se establecen unos vínculos emocionales muy profundos con la misma (Sanjuán, 2014). Las emociones que se han gestado de la relación con la literatura (ya sean positivas ya sean negativas), quedan adheridas hondamente en los lectores afectando, con posterioridad, a sus preferencias y gustos. Sirven, asimismo, para reafirmar que el conocimiento es ingénito a la emoción y a la afectividad (Krathwohl, Bloom y Masia, 1964).

No esperábamos, sin embargo, que el recuerdo lector oral del docente no redundara también en esa cicatriz cerebral gestora de lectores ávidos. Es posible que, nuevamente, lo académico haga acto de presencia y que cualquier recuerdo relacionado con al ámbito escolar sea considerado como maldito por las connotaciones señaladas con anterioridad y por los usos instrumentales de la literatura. Las grandes expectativas generadas en torno a la lectura en voz alta por parte de los docentes hacen que se esperen resultados mayores, al menos significativos. Pero la muestra estudiada no constata las hipótesis creadas inicialmente.

Otro de los datos extraídos resulta reconfortante. Los porcentajes apuntan a que los lectores que demuestran de forma temprana gusto por la lectura (basándonos en las lecturas placenteras), se mantienen como tales durante tiempo, convirtiéndose en lectores habituales o empedernidos. Los resultados no vienen más que a reincidir, una vez más, en la necesidad de educar literariamente desde la infancia con la finalidad de dejar buenos recuerdos y despertar las emociones necesarias, que permitan desarrollar y mantener el hábito lector.

Bastante significativo resulta que el poso de la emoción afecte por igual a hombres y mujeres, dato que, por extensión, vendría a demostrar dos hechos. El primero de ellos, que la emotividad no es un rasgo intrínseco y exclusivo del género femenino, sino más bien una conducta aprendida o impuesta socialmente. Y, en segundo lugar, que quizá no ha lugar a continuar con la clásica querelle sobre la existencia de un cerebro sexuado, puesto que vemos que su funcionamiento, al 
menos en lo que a influencia de la emoción se refiere, es idéntico, independientemente del género al que se pertenezca.

Cabe hacer referencia, antes de concluir, a algunas de las limitaciones de este estudio. Convendría ampliar la muestra de sujetos añadiendo otros centros de formación de maestros ajenos a la Universidad de Valladolid. Quizá, también sería productivo, poder realizar un estudio longitudinal que analizara la trayectoria de los encuestados. Finalmente, se podrían ampliar las preguntas relativas a la frecuencia lectora indagando sobre el número de libros que leen al año y la clase de los mismos. En todo caso, el camino está iniciado.

Sí se debería reincidir, por el análisis de los datos extraídos de esta investigación, en la importancia que adquieren las emociones infantiles provocadas por la lectura y sus aledaños. Es decir, por los libros, los espacios, las sensaciones y los adultos que formaron parte de nuestro ecosistema lector y que contribuyeron a desarrollarlo. Emoción y recuerdo son piezas indisolubles de los intertextos lectores desde la infancia y a lo largo de la vida. Leer y amar la lectura lleva impregnado el olor de todo aquello que nos ha acompañado. El hábito lector se ve estimulado desde edades tempranas y se desarrolla y asienta con un mayor vigor cuanto mejor es el recuerdo y por ende la emoción.

\section{Referencias bibliográficas}

Applegate, A. J., y Applegate, M. D. (2004). The Peter Effect: Reading habits and attitudes of preservice teachers. The Reading Teacher, 57(6), 554-563.

Barton, D., y Hamilton, M. (1998). Vernacular Practices. En D. Barton, y M. Hamilton, Local literacies. Reading and writing in one community (pp. 247-262). Londres/Nueva York: Routledge.

Bayard, P. (2008). Cómo hablar de los libros que no se han leído. Barcelona: Anagrama.

Benevides, T., y Peterson, S. S. (2010). Literacy attitudes, habits and achievements of future teachers, Journal of Education for 
Teaching, $36(3)$ 291-302.

doi: https://doi.org/10.1080/02607476.2010.497375.

Bettleheim, B., y Zelan, K. (2001). Aprender a leer. Barcelona: Crítica.

Beuchat, C. (2013). ¿Por qué leer en voz alta? Están los libros, están los niños... Consideraciones sobre la lectura en voz alta. En $A$ viva voz. Lectura en voz alta (pp. 16-27). Santiago de Chile: Ministerio de Educación de Chile.

Bian, L., Leslie, S. J., y Cimpian, A. (2017). Gender stereotypes about intellectual ability emerge early and influence children's interests. Science, 355(6323), 389-391. doi: https://doi.org/10.1126/science.aah6524.

Caivano, F. (2001). Los nuevos lectores del siglo XXI. Lector y lectura de calidad. En La educación lectora. Encuentro Iberoamericano (pp. 181-198). Madrid: Fundación Germán Sánchez Ruipérez.

Camitta, M. (1993). Vernacular writing: Varieties of Literacy among Philadelphia High School Student. En B. Street (Ed.). CrossCultural Approaches to Literacy (pp. 228-246). Cambridge: Cambridge University Press.

Carlino, P. (2006). Escribir, leer y aprender en la universidad. Una introducción a la alfabetización académica. Buenos Aires: Fondo de Cultura Económica.

Chartier, R., y Bourdieu, P. (1985). La lecture: une pratique culturelle. Débat entre Pierre Bourdieu et Roger Chartier. En R. Chartier (Ed.). Pratiques de la lecture (pp. 266-294). Paris, Éditions Rivages.

Colomer, T., y Munita, F. (2013). La experiencia lectora de los alumnos de magisterio: nuevos desafíos para la formación docente. Lenguaje y Textos, 38, 37-45.

Cremin, T., Bearne, E., Mottram, M., y Goodwin, P. (2008). Primary teachers as readers. English in Education, 42(1), 8-23.

Damasio, A. (1999). The feeling of what happens. Londres: William Heinemann.

Devetach, Laura (2008). La construcción del camino lector. Córdoba: Comunicarte. 
Díaz-Plaja, A., y Prats, M. (2013). Recordar, aprender, practicar: qué sabe y qué debe saber un futuro maestro. Lenguaje y Textos, 38, 19-28.

Farrugia, P., Petrisor, B., Farrokhyar, F., y Bhandari, M. (2010). Research questions, hypotheses and objectives. Canadian Journal of Surgery, 53(4), 278-281.

Fox, M. (2008). Why Reading Aloud to our Children will Change their Lives Forever. Nueva York: Harcourt

Granado. C. (2013). La formación inicial de maestros y maestras en educación lectora: perspectiva del alumnado y contenidos ofrecidos en tres universidades andaluzas. Investigación en la escuela, 80, 103-115. doi: https://doi.org/10.12795/IE.2013.i80.08

Granado, C. (2014). El docente como lector: estudio de los hábitos lectores de futuros maestros. Culture and Education, 26(1), 4470. doi: https://doi.org/10.1080/11356405.2014.908666.

Granado, C., y Puig, M. (2015). La identidad lectora de los maestros en formación como componente de su identidad docente. Un estudio de sus autobiografías como lectores. Ocnos: Revista de Estudios sobre Lectura, 13 , 43-63. doi: https://doi.org/10.18239/ocnos_2015.13.03.

Gupta, R. (2004). Old habits die hard: Literacy practices of preservice teachers. Journal of Education for Teaching, 30(1), 67-78. doi: https://doi.org/10.1080/0260747032000162325.

James, W. (1884). What is an emotion? Mind, 9 (34), 188-205.

Krathwohl, D. R., Bloom, B. S., y Masia, B. B. (Eds.). (1964). Taxonomy of educational objectives: Handbook II: The affective domain. Nueva York: McKay.

Le Doux, J. (1999). El Cerebro Emocional. Barcelona: Ariel/Planeta.

Lea, M. R., y Street, B. V. (2006). The "Academic Literacies" Model: Theory and Applications. Theory Into Practice, 45(4), 368-377. Doi: https://doi.org/10.1207/s15430421tip4504_11.

Marañón, G. (1920). La emoción. Revista voluntad, IX, s. p. Recuperado

de: http://hemerotecadigital.bne.es/issue.vm?id=0003823897\&search=\&lan $\mathrm{g}=\mathrm{es}$. 
Martínez, M. M., y Torres, A. (2019). Hábito lector en estudiantes de primaria: influencia familiar y del plan lector del centro escolar. Revista Fuentes, 21(1), 103-114. doi: https://doi.org/10.12795/revistafuentes.2019.v21.i1.07.

Mata, J. (2010), La educación como lectura. En A. Basanta (Coord.). La lectura (pp. 103-119). Madrid: CSIC/Catarata.

Mendoza, A., y Díaz, A. (2005). De l'enseignement de la littérature dans le cadre scolaire espagnol à la dormation pour l'éducation littéraire. Repères. Recherches en didactique du français langue maternelle, 32, 159-192.

Ministerio de Educación y Formación Profesional (2019). Igualdad en cifras MEFP. Madrid: Secretaría General Técnica, Subdirección General de Atención al Ciudadano, Documentación y Publicaciones.

Mora, F. (2013a). ¿Qué es una emoción? Arbor Ciencia, Pensamiento y Cultura, 189(759), 1-6. doi: http://dx.doi.org/10.3989/arbor.2013.759n1003.

Mora, F. (2013b). Neuroeducación. Solo se puede aprender aquello que se ama. Madrid: Alianza Editorial.

Mora, F. (2020). Neuroeducación y lectura. De la emoción a la comprensión de las palabras. Madrid: Alianza Editorial.

Mora-Figueroa, J., Galán, A., y López-Jurado, M. (2016). Efectos de la implicación familiar en estudiantes con riesgo de dificultad lectora. Ocnos: Revista de estudios sobre lectura, 15(1), 7-21. doi: https//:doi.org/10.18239/ocnos_2016.15.1.866

Munita, F. (2012). Perfil lector del futuro profesorado: Discurso y prácticas de los formadores de lectores. En V. Ruiz y I. Plazaola (Eds.). Actas del $V$ Seminario El aula como ámbito de investigación sobre la enseñanza y aprendizaje de la lengua (pp. 189200). Bizkaia: Servicio de Publicaciones del País Vasco.

Pellowski, A. (1987). The Family Storytelling Handbook. Nueva York: Macmillan Publishing Co.

Piscitelli, A. (2002). Ciberculturas 2.0. En la era de las máquinas inteligentes. Buenos Aires: Paidós, Contextos.

Ribera, P., y Romero, M. F. (2020). El adolescente como lector accidental de textos literarios. Hábitos de lectura literaria en Educación 
Secundaria. Ocnos: Revista de Estudios sobre Lectura, 19(3), 7-18. doi: https://doi.org/10.18239/ocnos_2020.19.3.2313.

Romero, F. (2007). Utopías lectoras y promoción de la lectura en la escuela. En Anuario sobre el libro infantil y juvenil 2007 (pp. 81114). Madrid: Ediciones SM.

Romero, M. F., y Álvarez, E. (2018). El cuento en las biografías lectoras de los más jóvenes y sus historias completas. En E. Álvarez (Ed.). Acción y efecto de contar. Estudios sobre el cuento hispánico contemporáneo (pp. 99-116). Madrid: Visor libros.

Routman, R. (2000). Conversations: Strategies for Teaching, Learning and Evaluating. Portsmouth, New Hampshire: Heinemann.

Sainsbury, M., y Schagen, I. (2004). Attitudes to reading at ages nine and eleven. Journal of Research in Reading, 27(4), 373-386. doi: https://doi.org/10.1111/j.1467-9817.2004.00240.x.

Salazar, S. (2005a). Claves para pensar la formación del hábito lector. Allpanchis, 37 (66), 13-46. doi: https://doi.org/10.36901/allpanchis.v37i66.492.

Sanjuán, M. (2011). De la experiencia de la lectura a la educación literaria. Análisis de los componentes emocionales de la lectura literaria en la infancia y la adolescencia. Ocnos: Revista de Estudios sobre Lectura, 7, 85-99. doi: https://doi.org/10.18239/ocnos_2011.07.07

Sanjuán, M. (2014). La dimensión emocional en la educación literaria. Zaragoza: Prensas de la Universidad de Zaragoza.

Silverman, D. (2013) Doing Qualitative Research: A Practical Handbook. Los Ángeles / Londres / Nueva Delhi / Singapur | Washington DC: SAGE Publications.

Smith, F. (1995). What happens when you read? En F. Smith, Between hope and havoc. Essays Into Human Learning and Education (pp. 27-39). Portsmouth: Heinemann.

Strommen, L., y Mates, B. (2004). Learning to Love Reading: Interviews With Older Children and Teens. Journal of Adolescent \& Adult Literacy, 48(3), 188-200.

Wigfield, A., y Guthrie, J. T. (1997). Relations of children's motivation for reading to the amount and breadth of their reading. Journal of Educational Psychology, 89(3), 420-432. 
Wilson, E. O. (1998). Consilience: The Unity of Knowledge. Nueva York: Alfred A. Knopf, Inc. 
Tejuelo, $\mathrm{n}^{\circ} 34$ (2021), págs. 293-322. El recuerdo y la emoción en la adquisición del bábito lector ... 